\title{
Initiation of renal replacement therapy in patients with sepsis: more to it than meets the eye
}

\author{
Fabrice Uhel, Hessel Peters-Sengers, Tom van der Poll \\ Center for Experimental and Molecular Medicine, Amsterdam University Medical Centers, Location Academic Medical Center, University of \\ Amsterdam, Amsterdam, The Netherlands \\ Correspondence to: Fabrice Uhel, MD, PhD. Center for Experimental and Molecular Medicine, Academic Medical Center, Room G2-130, \\ Meibergdreef 9, 1105 AZ Amsterdam, The Netherlands. Email: f.uhel@amc.uva.nl. \\ Provenance: This is an invited Editorial commissioned by Section Editor Wei Liu, MD (Department of Nephrology, The Affiliated Anqing Hospital \\ of Anhui Medical University, Anqing, China). \\ Comment on: Barbar SD, Clere-Jehl R, Bourredjem A, et al. Timing of Renal-Replacement Therapy in Patients with Acute Kidney Injury and Sepsis. \\ N Engl J Med 2018;379:1431-42.
}

Submitted Dec 11, 2018. Accepted for publication Dec 14, 2018.

doi: $10.21037 / \mathrm{atm} .2018 .12 .36$

View this article at: http://dx.doi.org/10.21037/atm.2018.12.36

If the host response to an infection becomes unbalanced and fails to return to normal homeostasis, it induces severe systemic disturbances and organ dysfunction, defining sepsis (1). Septic shock is the most severe presentation of this syndrome, characterized by circulatory, cellular, and metabolic abnormalities requiring hemodynamic support by vasopressors. This context frequently features an impairment of renal function, defined as septic acute kidney injury (AKI) (2). In the intensive care unit (ICU), sepsis accounts for approximately half of all causes of AKI. The incidence of AKI during sepsis increases from $20 \%$ to $50 \%$, parallel to severity, and is an independent contributor to mortality (3). The diagnosis of AKI relies on relative changes in serum creatinine and urine output. The Risk Injury Failure Loss End-stage renal disease (RIFLE) criteria were initially proposed by the acute dialysis quality initiative to define and classify acute changes in renal function (4). More recently, a consensual definition has been released by the Kidney Disease Improving Global Outcomes (KDIGO) group (5).

The pathophysiology of septic AKI remains only partially understood. Recent advances have challenged the longstanding theory of acute tubular necrosis secondary to a global kidney hypoperfusion. Both human postmortem histopathological studies and experimental studies in sheep revealed minimal structural kidney lesions despite clinically severe AKI. Besides, animal studies showed that septic AKI, at least at the early phase, might mainly involve functional changes, including microvascular shunting and tubular cell stress $(6,7)$. These data suggest that early interventions could improve kidney function and prevent persistence of AKI.

Unfortunately, there is currently no pharmacological treatment that is able to induce renal repair in patients with established septic AKI. Current standard of care relies on treatment of the infection, adequate hemodynamic resuscitation, and avoidance of further nephrotoxic insults. If renal function becomes insufficient to maintain physiological roles of kidneys, support by renal replacement therapy (RRT) becomes necessary. Because septic patients receiving RRT are among the more severely ill in the ICU, the optimal management of this therapy is of utmost importance. If the optimal intensity and modalities have been well established, deciding when to initiate RRT remains a particularly challenging question.

In the situation of life-threatening electrolyte, acid-base or fluid imbalance, the indication for RRT is indisputable and the procedure should be initiated without delay. However, most critically ill patients with AKI do not have such an absolute indication for RRT. In all other clinical scenarios, the appropriate timing of the initiation of RRT remains unclear. While the KDIGO guidelines leave this to the opinion of the treating physician considering clinical and biological contexts, the Surviving Sepsis Campaign Guidelines suggest not using RRT for increased creatinine or oliguria alone without other definitive indications for dialysis $(5,8)$.

Two strategies compete in the process of clinical decision, 
an early strategy based on the rapid initiation of RRT after reaching a certain AKI severity, versus a delayed "wait-andsee" strategy, where RRT is started only in the absence of renal function recovery or if AKI-related complications occur. Both strategies have their own advantages and pitfalls. On the one hand, early initiation of RRT may rapidly improve and stabilize acid-base, electrolyte and fluid balance, thereby preventing more severe complications of AKI and possibly remove potentially toxic substances. However, such a strategy implies the unnecessary initiation of RRT and exposure to risks associated with catheters and extracorporeal circulation in a subset of patients in whom renal function would have recovered spontaneously. Besides, early initiation of RRT might be difficult at the early phase of sepsis in hemodynamically unstable patients and may impact the adequate dosing of antibiotic therapy (9). On the other hand, a delayed strategy for the initiation of RRT could allow time for stabilization of the patient's situation, thus enabling starting of RRT in better hemodynamic conditions, or even obviating the need for such therapy if the renal function rapidly recovers. However, this approach also entails risks linked to prolonged exposure to AKI-induced complications, such as metabolic disorders or fluid overload.

Several studies have attempted to provide an answer to this dilemma. A meta-analysis, mostly derived from observational studies, suggested a reduction in 28-day mortality in favor of earlier starts (10). However, the strength of the conclusion was weakened by the heterogeneous definition of "early RRT” across studies. Two randomized control trials (RCTs) published in 2016 comparing different timing strategies for the initiation of RRT in general populations of critically ill patients reported conflicting results. The single-center ELAIN study of 231 surgical patients showed a reduction in day-90 mortality when RRT was initiated at KDIGO stage-2 AKI rather than waiting for the onset of a KDIGO stage-3 AKI (11). In contrast, in the multicenter AKIKI study including 620 patients from mainly medical ICUs with more severe KDIGO stage-3 AKI, the delayed "wait-andsee" strategy could avoid RRT in $49 \%$ of patients and did not show any difference in day-60 mortality (12). In the more specific context of sepsis, a post hoc analysis of the AKIKI study showed that early RRT-initiation strategy was not associated with any improvement of day-60 mortality and could even delay renal function recovery (13). Consistently, a smaller RCT had also shown that the early application of RRT could be harmful and increase the number and severity of organ failures (14).

In a recent issue of the New England Fournal of Medicine,
Barbar and colleagues published the results of the IDEALICU study, focusing on the outcome of patients with a severe AKI in the context of septic shock (15). This RCT conducted in 29 mixed medical and surgical ICUs in France compared two strategies for the initiation of RRT. In the early strategy group (246 patients), patients received RRT within 12 hours following the diagnosis of RIFLE-F AKI (equivalent to $\mathrm{KDIGO}$ stage 3 : creatinine $\geq 3$ times baseline or $\geq 4 \mathrm{mg} / \mathrm{dL}$, urine output $<0.3 \mathrm{~mL} / \mathrm{kg} / \mathrm{h}$ for 24 hours, or anuria for 12 hours). In the delayed-strategy group (242 patients), initiation of RRT was postponed by 48 hours after documentation of RIFLE-F AKI, except if the patient required earlier RRT for critical criteria (hyperkaliemia, severe acidosis or pulmonary edema secondary to fluid overload and refractory to diuretics). If renal function recovered spontaneously, RRT was not initiated. The study was stopped for futility after the second interim analysis, including 488 patients of the 864 initially planned. Indeed, the independent data and safety monitoring board considered it unlikely that further enrolment of patients would change the results obtained. In the intention-totreat analysis, 90-day mortality (predefined as the primary endpoint of the trial) was $58 \%$ in the early strategy group, versus $54 \%$ in the late-strategy group. No differences were either found regarding secondary outcomes-days free from mechanical ventilation, ICU or hospital length of stay. The two strategies were not associated with differences in the course of sepsis (similar number of vasopressor-free days, evolution of non-renal SOFA), the course of AKI (no difference in the dependence on RRT among survivors at day 28), or the occurrence of ICU-acquired complications (secondary infections and thrombo-embolic complications).

Even if a new negative trial might appear disappointing at first sight, a deeper look at details underlines insightful information. We must first acknowledge the quality of the study design, which included a clinically well-defined population of patients with septic shock and severe RIFLE-F AKI, hence with a high a priori risk of requiring RRT. However, the "wait-and-see" strategy, which is probably close to the current standard of care, interestingly revealed an important heterogeneity in terms of evolution and outcomes. Indeed, almost 1 in 3 patients (29\%) in the delayed strategy group recovered spontaneously and didn't require RRT, which presumes that a similar proportion of patients received RRT unnecessarily in the early strategy group, thereby causing pointless exposure to possible catheter and extracorporeal circulation-associated complications, not to mention time, personnel and financial 
costs. Of note, mortality was twice as low in this subgroup of patients $(26 \%)$. In contrast, 41 patients $(17 \%)$ in the delayed strategy group required emergency RRT before 48 hours after the diagnosis of AKI because of life threatening complications (severe metabolic acidosis, hyperkaliemia or fluid overload). A total of 28 (68\%) of these patients died, highlighting the particular severity of this subgroup, or the increased risk of mortality attributable to the occurrence of such complications. Unfortunately, it was not reported how these subgroups of patients differed at baseline.

Although results from subgroup analyses should be considered carefully, they still underline two major points: (I) the RIFLE score failed to accurately identify patients who went on to require RRT; (II) both early and delayed therapeutic strategies had various impacts on different subgroups of patients. These points raise the following question: is there a way to better predict which patients are likely to require RRT (because of the occurrence of a critical indication, or because of the absence of recovery) and which patients have a high likelihood of spontaneous recovery?

This question underpins the main purpose of predictive enrichment strategies, which aim at identifying patient subsets who are more likely to benefit from a specific therapeutic intervention, in order to inform future trial designs (16). Different methods could be used to achieve predictive enrichment for requirement of RRT. The first approach relies on the use of biomarkers. Plasma cystatin $\mathrm{C}$ or neutrophil gelatinase-associated lipocalin (NGAL) have shown reasonable prediction of RRT use in critically ill patients with AKI. Yet, the still low strength of evidence precludes their routine use pending further validation (17). The discovery of new biomarkers is thus urgently needed to identify patients who will have a disease progression and definitely require RRT.

Another approach is the use of empiric algorithms in order to group patients based on their clinical and biological characteristics. Using such approaches, recent studies have underlined the complex heterogeneity of patients with sepsis at the level of blood leukocyte molecular responses $(18,19)$. Recently, Calfee and colleagues reanalyzed data from four RCTs in patients with acute respiratory distress syndrome (ARDS) (20-22). Using latent class analyses, they identified two subphenotypes, one of which was characterized by more severe inflammation, shock, and metabolic acidosis and by worse clinical outcomes. Interestingly, there was a significant interaction with the treatment tested in the RCTs (positive end-expiratory pressure, fluid management, or simvastatin), suggesting that these subphenotypes could identify patients likely to show different responses to the therapeutic intervention tested.

The study by Barbar and colleagues is an important new contribution to current knowledge on the timing of initiation of RRT in patients with AKI in the context of septic shock. The negative result on the primary endpoint turns out to hide a high level of heterogeneity in terms of disease progression, that could not be accurately predicted by the staging of AKI at time of inclusion. It also strongly suggests that one size doesn't fit all, and that both early and delayed approaches could bring variable levels of benefit to specific subgroups of patients. The challenge is now to better identify, using clinical or biological profiles, patients who are more likely to benefit from each approach, and tailor future trials based on risk-stratification strategies. Similar to sepsis and ARDS, the time has come for AKI to go deeper into the field of personalized medicine.

\section{Acknowledgements}

None.

\section{Footnote}

Conflicts of Interest: The authors have no conflicts of interest to declare.

\section{References}

1. van der Poll T, van de Veerdonk FL, Scicluna BP, et al. The immunopathology of sepsis and potential therapeutic targets. Nat Rev Immunol 2017;17:407-20.

2. Bellomo R, Kellum JA, Ronco C, et al. Acute kidney injury in sepsis. Intensive Care Med 2017;43:816-28.

3. Hoste EAJ, Kellum JA, Selby NM, et al. Global epidemiology and outcomes of acute kidney injury. Nat Rev Nephrol 2018;14:607-25.

4. Bellomo R, Ronco C, Kellum JA, et al. Acute renal failure definition, outcome measures, animal models, fluid therapy and information technology needs: the Second International Consensus Conference of the Acute Dialysis Quality Initiative (ADQI) Group. Crit Care 2004;8:R204-12.

5. KDIGO AKI Work Group. KDIGO Clinical Practice Guideline for Acute Kidney Injury. Kidney Int Suppl 2012;2:1-141.

6. Calzavacca P, Evans RG, Bailey M, et al. Cortical and Medullary Tissue Perfusion and Oxygenation in Experimental Septic Acute Kidney Injury. Crit Care Med 
2015;43:e431-9.

7. Mariano F, Cantaluppi V, Stella M, et al. Circulating plasma factors induce tubular and glomerular alterations in septic burns patients. Crit Care 2008;12:R42.

8. Rhodes A, Evans LE, Alhazzani W, et al. Surviving Sepsis Campaign: International Guidelines for Management of Sepsis and Septic Shock: 2016. Crit Care Med 2017;45:486-552.

9. Roberts DM, Liu X, Roberts JA, et al. A multicenter study on the effect of continuous hemodiafiltration intensity on antibiotic pharmacokinetics. Crit Care 2015;19:84.

10. Karvellas CJ, Farhat MR, Sajjad I, et al. A comparison of early versus late initiation of renal replacement therapy in critically ill patients with acute kidney injury: a systematic review and meta-analysis. Crit Care 2011;15:R72.

11. Zarbock A, Kellum JA, Schmidt C, et al. Effect of Early vs Delayed Initiation of Renal Replacement Therapy on Mortality in Critically Ill Patients With Acute Kidney Injury: The ELAIN Randomized Clinical Trial. JAMA 2016;315:2190-9.

12. Gaudry S, Hajage D, Schortgen F, et al. Initiation Strategies for Renal-Replacement Therapy in the Intensive Care Unit. N Engl J Med 2016;375:122-33.

13. Gaudry S, Hajage D, Schortgen F, et al. Timing of Renal Support and Outcome of Septic Shock and Acute Respiratory Distress Syndrome. A Post Hoc Analysis of the AKIKI Randomized Clinical Trial. Am J Respir Crit Care Med 2018;198:58-66.

14. Payen D, Mateo J, Cavaillon JM, et al. Impact of continuous venovenous hemofiltration on organ failure during the early phase of severe sepsis: a randomized

Cite this article as: Uhel F, Peters-Sengers H, van der Poll T. Initiation of renal replacement therapy in patients with sepsis: more to it than meets the eye. Ann Transl Med 2018;6(Suppl 2):S130. doi: 10.21037/atm.2018.12.36 controlled trial. Crit Care Med 2009;37:803-10.

15. Barbar SD, Clere-Jehl R, Bourredjem A, et al. Timing of Renal-Replacement Therapy in Patients with Acute Kidney Injury and Sepsis. N Engl J Med 2018;379:1431-42.

16. Shankar-Hari M, Rubenfeld GD. The use of enrichment to reduce statistically indeterminate or negative trials in critical care. Anaesthesia 2017;72:560-5.

17. Klein SJ, Brandtner AK, Lehner GF, et al. Biomarkers for prediction of renal replacement therapy in acute kidney injury: a systematic review and meta-analysis. Intensive Care Med 2018;44:323-36.

18. Scicluna BP, van Vught LA, Zwinderman AH, et al. Classification of patients with sepsis according to blood genomic endotype: a prospective cohort study. Lancet Respir Med 2017;5:816-26.

19. Davenport EE, Burnham KL, Radhakrishnan J, et al. Genomic landscape of the individual host response and outcomes in sepsis: a prospective cohort study. Lancet Respir Med 2016;4:259-71.

20. Calfee CS, Delucchi K, Parsons PE, et al. Subphenotypes in acute respiratory distress syndrome: latent class analysis of data from two randomised controlled trials. Lancet Respir Med 2014;2:611-20.

21. Famous KR, Delucchi K, Ware LB, et al. Acute Respiratory Distress Syndrome Subphenotypes Respond Differently to Randomized Fluid Management Strategy. Am J Respir Crit Care Med 2017;195:331-8.

22. Calfee CS, Delucchi KL, Sinha P, et al. Acute respiratory distress syndrome subphenotypes and differential response to simvastatin: secondary analysis of a randomised controlled trial. Lancet Respir Med 2018;6:691-8. 